\title{
Essays on OR in ALIO country members (part 2)
}

\author{
Laureano F. Escudero $^{1} \cdot$ Antonio Alonso-Ayuso ${ }^{1} \cdot$ Nelson Maculan $^{2}$
}

Received: 6 February 2021 / Accepted: 6 February 2021 / Published online: 17 March 2021

(c) Sociedad de Estadística e Investigación Operativa 2021

Mathematic Subject Classification 90B90 · 90B06 · 90C06 · 90C08 · 90C46 · 90C90

The Spanish Sociedad de Estadística e Investigación-Operativa, SEIO, devotes two volumes of its journal TOP (co-guest-edited by Antonio Alonso-Ayuso, Laureano F. Escudero and Nelson Maculan) to present works by representative authors of ALIO member countries. ALIO (Asociación Latino-Iberoamericana de InvestigaciónOperativa), https://www.alio-online.org/, is a regional association within IFORS. This volume is the second of the two ones, where selected peer-reviewed works are collected. Those works vary from a mixture of more theoretical and exact and heuristic algorithmic developments in capacitated location routing problems, the new ordered median tree of the hub location problem and shortest alpha-reliable path problems to stochastic dynamic programming for long-term optimal gas supply and storage, stochastic portfolio selection and stochastic online optimization of airplane seat assignment, multi-objective optimization for getting a trade-off among aims that go in different directions, sport scheduling, food bank allocation, and zoning crop planning via column-based generation formulations.

The aims of the works are as follows:

Classification and regression trees are studied in the work "Mathematical optimization in classification and regression trees" by Emilio Carrizosa, Cristina MoleroRío and Dolores Romero Morales. Those trees and their variants are analyzed, and recent contributions are reviewed within the Continuous Optimization and the Mixed-Integer Linear Optimization paradigms to develop novel formulations in this research area. Some algorithms are proposed and compared. The formulations that have been contributed are suitable for incorporating desirable properties such as cost

Laureano F. Escudero

laureano.escudero@urjc.es

Antonio Alonso-Ayuso

antonio.alonso@urjc.es

Nelson Maculan

maculan@cos.ufrj.br

1 Universidad Rey Juan Carlos, Madrid, Spain

2 Universidade Federal de Rio de Janeiro, Rio de Janeiro, Brazil 
sensitivity, explainability and fairness, and to deal with complex data, such as functional data.

The work "A Branch \& Cut algorithm for the prize-collecting capacitated location routing problem" by Daniel Negrotto and Irene Loisseau addresses the capacitated location routing problem (CLRP) as the combination of two well-studied problems in OR, namely, the capacitated facility location problem (CFLP) and the multiple depots vehicle routing problem (MDVRP). Given a set of available locations and a fleet of vehicles, the aim is to determine a set of depots to open and the routes of the vehicles to satisfy the customer demands. The prize-collecting capacitated location routing problem (PC-CLRP) is presented as new variant of the CLRP. For problem solving a two-index formulation for the PC-CLRP and a Branch \& Cut algorithm are proposed jointly with a new set of valid inequalities and optimality cuts jointly with their corresponding separation algorithms.

Marina Baltar, Victor Abreu, Glaydston Ribeiro and Laura Bahiense explore the trade-off between the maximization of traffic incident attendance and the minimization of $\mathrm{CO} 2$ emissions addressed in their contribution "Multi-objective model for the problem of locating tows for incident servicing on expressways". A real-world case study from Avenida Brasil, the main expressway of Rio de Janeiro, Brazil, is considered, and a mathematical multi-objective model is introduced, including queuing theory to locate tows for incident servicing. It is applied to 3080 real incidents, occurred between March and June of 2018, and the results showed that when the maximum response time is restricted, the quantity of tows has to be raised to make sure that there is an adequate servicing.

Miguel A. Pozo, Justo Puerto and Antonio M. Rodríguez Chía propose in their work "The ordered median tree of hubs location problem" the Ordered Median Tree of Hub Location Problem (OMTHL). It is a single-allocation hub location problem where $p$ hubs must be placed on a network and connected by a non-directed tree. Each non-hub node is assigned to a single hub and all the flow between origin-destination pairs must cross one hub, at least. Different MILP formulations are considered based on the properties of the minimum spanning tree problem, the ordered median optimization and on the different ways of modeling flow within the network. An empirical comparison is established between these formulations, where some enhancements are also provided, to solve medium-size instances on general random graphs.

Larissa de Oliveira Resende, Davi Valladão, Bernardo Vieira Bezerra and Yasmin Monteiro Cyrillo propose in their work "Assessing the value of natural gas underground storage in the Brazilian system via stochastic dual dynamic programming" a stochastic dynamic programming model for long/medium-term operation planning to determine the optimal gas supply and storage policies. That Markovian model characterizes the uncertainty over the thermoelectric demand and market price. It covers the lack in the existing literature of a proper analytical tool to assess the benefits of underground natural gas storage activity. The numerical simulations show that the economic benefit for the system surpasses the operational and capital expenses for the storage infrastructure in depleted fields and salt caverns.

Guillermo Durán presents an overview of OR tools for better decision making in sports in his work "Sports scheduling and other topics in sports analytics: a survey 
with special reference to Latin America". Known as sports scheduling, this field is one of the subjects of the survey, which reviews different problems tackled in the associated literature, the techniques employed for solving them and the results of their implementation in real-world cases. Other topics in sports analytics such as player performance, result prediction, fantasy games and analysis of rankings are also examined. Special attention is given to applications in Latin America. The main challenges facing sports scheduling and other areas of sports analytics are also discussed.

Portfolio selection problems have been thoroughly studied under the risk-andreturn paradigm introduced by Markowitz. In the work "The effect of regularization in portfolio selection problems", Bernardo Pagnoncelli, Felipe del Canto, and Arturo Cifuentes study the practical considerations that have resulted in poorly diversified portfolios, or solutions that are extremely sensitive to parameter estimation errors. For that purpose, sampling methods are considered to cope with this issue and compare the merits of two approaches, namely, a sample average approximation one and an extended performance-based regularization (PBR) method that appeared recently in the literature. The PBR extension considers different risk metrics. A numerical comparison using index-based portfolios is presented using historic data that includes the subprime crisis.

I. Gimenez-Palacios, María Teresa Alonso, Ramón Alvarez-Valdes and Francisco Parreño focus on their work "Logistic constraints in container loading problems: the impact of complete shipment conditions" on a special type of logistic constraints in the container loading problem (CLP) as it is the complete shipment requirements, which ensure that either all or none of the boxes in each customer order are loaded. Those constraints are included in an integer linear formulation of the CLP and four heuristic strategies are developed to deal with them efficiently. An extensive computational study compares the relative performance of these strategies and shows that when a good strategy is used the impact of these constraints on the solution of the CLP is quite small.

Jordi Castro and Fernando Sarachaga present in their work "An online optimization-based procedure for the assignment of airplane seats" a novel model for the seat assignment problem, which focuses on deciding where to seat the passengers of different online purchases. The approach is based on solving an integer multicommodity network flow problem, where different commodities are associated with expected future demands of different types of passengers. The usefulness of the model that is proposed requires to solve it in real time. Using a real database of flights by Vueling Airlines, a set of synthetic online purchases is simulated for a pseudo-real flight. CPLEX solver is used in real-time and, as a result, the seat assignment obtained was of higher quality than that obtained by the simple pre-defined rules used by airlines.

Victor M. Albornoz and Gabriel E. Zamora consider in their work "Decomposition-based heuristic for the zoning and crop planning problem with adjacency constraints" the management zone delineation and crop planning problems in an integrated agriculture framework. The zoning problem defines relatively homogeneous management zones regarding their soil properties. A novel linear binary integer program is proposed. It is a column-based formulation and, as such, a decompositionbased heuristic is proposed which make use of the column generation method with 
column-dependent rows. The decomposition strategy involves a master problem that deals with ensuring homogeneity of the selected management zones within the field partition and ensuring that the crop plan meets adjacency policies. The algorithm is implemented in JuMP, a modeling language for mathematical optimization embedded in Julia. Results from a set of instances show the relevance of the decomposition-based heuristic.

One of the global strategic areas in the fight against hunger is the one related to food banks. The mission of food banks is to provide food to people who are in extreme poverty and famine. However, food banks do not have enough resources to supply food to the needy. Hence, hard decisions have to be made every day to determine who will be served, what kind of products, and how many of them will be supplied. In the work "The food bank resource allocation problem" J. Alvaro Gómez-Pantoja, Angélica Salazar-Aguilar, José Luis González-Velarde introduce an optimization model for the Food Bank Resource Allocation Problem, which takes into account inventory management, purchases, product-beneficiary compatibilities, balanced nutrition, and priority of beneficiaries. An adaptive heuristic is proposed to solve large instances of this problem. Computational results reveal that it is able to produce good quality solutions in short computation times.

In "On the Shortest alpha-Reliable Path Problem", David Corredor-Montenegro, Nicolás Cabrera, Raha Akhavan-Tabatabaei and Andrés L. Medaglia extend the pulse algorithm for solving a variant of the constrained shortest path problem. It consists of finding a minimum cost path from an origin to a destination, ensuring that the probability of reaching the destination within a time limit meets a certain reliability threshold. The random variables of the travel times are modeled using phase-type distributions and Monte Carlo simulation. A set of experiments over small (and medium) size stochastic transportation networks are carried out with and without spatially correlated travel times. As an alternative to handling correlations, a scenario-based approach is presented where the distributions of the arc travel times are conditioned to a given scenario (e.g., variable weather conditions). The proposed methodology and the experiments that have been conducted highlight the relevance of considering on-time arrival probabilities and correlations when solving shortest path problems.

The Guest-coEditors like to express their most sincere gratitude to the former editors of TOP, Gustavo Bergantiños, and Juan José Salazar, for giving them the opportunity of preparing this special issue, and to the authors for the effort that have been made during these two years.

Publisher's Note Springer Nature remains neutral with regard to jurisdictional claims in published maps and institutional affiliations. 